\title{
Investigation of Factors Affecting Extraction of Rebaudioside A \& Stevioside from Stevia Leaves
}

\author{
Kalaiarasan Muthusamy ${ }^{1}$, Mimi Sakinah Abdul Munaim ${ }^{2 *}$ \\ 1,2* Faculty of Engineering Technology, Universiti Malaysia Pahang, 26300 Gambang, Pahang, Malaysia
}

*Email: kalaiarasanmuthusamy1995@gmail.com,mimi@ump.edu.my

\begin{abstract}
Stevia is a sugar substitute extricated from the leaves of Stevia rebaudiana, found abundantly in Brazil and Paraguay. The active compounds are steviol glycosides (stevioside and rebaudioside), which are 30 to 150 times sweeter than sugar, heat and $\mathrm{pH}$ resistant and not fermentable. The extensive use of sugar is becoming dangerous by causing lot of health complications, especially diabetes. Therefore, a substitute sweetener has to be produced to maintain consumers' health condition. Solidliquid extraction is a recovery process in which the extracting solvent will recover certain components from a solid material. In this study, three extracting solvents were used which are absolute ethanol, acetone and distilled water. These solvents extracted two sweet components, Rebaudioside A and stevioside from Stevia rebaudiana. These two components can be converted into natural sweetener with zero calorie which does not affect blood glucose level. The procedure for this process can be divided into 3 phases. First, preparation of powdered stevia and extraction of Rebaudioside A and stevioside using ethanol, acetone and water. Next, centrifugation of liquid phase and filtration of supernatant from precipitate. Finally, HPLC analysis where the chromatogram of each supernatant sample was compared to the standard to obtain retention time and peak areas of Rebaudioside A and stevioside. From this study, it is proven that ethanol is the optimum extraction agent. The optimized condition for ethanol extraction of Rebaudioside A and stevioside was ratio $1: 25,1$ hour and $40^{\circ} \mathrm{C}$ which produced $12.48 \%$ of stevioside and $0.57 \%$ of Rebaudioside A.
\end{abstract}

Keywords: Material ratio, temperature, time, Rebaudioside A, stevioside

\section{INTRODUCTION}

The expanding usage of sugar has brought about a few nutritional and medical issues such as obesity which leads to diabetics and eventually cardiovascular diseases with more attention on dental caries issue. Increase in the number of the diseases can be related to uncontrolled intake of glucose and highcalorie sweeteners [13]. World Health Organization (WHO) detailed that 371 million individuals worldwide have diabetes in 2011. The assessed number of diabetics increased in 2014 to 422 million. There are around 1.6 million people passed away due to diabetes in 2016 [3]. Each year, the number of diabetic patients builds quickly and studies in 2017 express that more than 425 million individuals worldwide have diabetes.

The food industry customarily uses table sugar as the main source of sweetening agent. However, there is an expanding interest among customers for Stevia-based natural source sweeteners [9]. Also, the sweetener is safe to be consumed, has almost zero calorie, unaffected by heat and $\mathrm{pH}$ changes and last but not least, tastes exactly like sugar. Stevia is a pleasant tasting herb and a member of the daisy family [10]. There are many types of stevia such as Stevia eupatoria, Stevia ovata, Stevia plummerae, Stevia rebaudiana, Stevia salicifolia and Stevia serrata. Stevia rebaudiana is one of the 154 members from the genus stevia and one of just two that produce sweet glycosides [19]. 
Numerous extraction methods have been tried and tested to extract steviol glycosides from stevia leaves. One of them include green extraction of glycosides using water and ethanol [12]. In this process, individual effects of drug powder size, weight ratio of drug to solvent, temperature, agitation, and time on the yield of the glycosides were evaluated. The results showed that ethanol $90 \%$ extracted more glycosides compared to water. Another one is subcritical water extraction of glycosides [12]. Subcritical water is water that is held by pressure at a temperature higher than its natural boiling point of $100^{\circ} \mathrm{C}$. The parameters investigated in this experiment were temperature, time and flow rate. Optimum extraction condition was determined as $125^{\circ} \mathrm{C}, 45 \mathrm{~min}, 4 \mathrm{ml} / \mathrm{min}$ flow rate which yielded $38.67 \mathrm{mg} / \mathrm{g}$ stevioside and $35.68 \mathrm{mg} / \mathrm{g}$ Reb A. Another method is ultrasonic extraction but this poses a risk of degradation of the glycosides [11].

Furthermore, extraction processes done before consume a tremendous amount of time with complex series of steps while causing hazardous effects. The lengthy extraction procedures of glycosides are capable of producing toxic residues and bitter alkaloids which are detrimental to human health [15]. Sometimes, reaction between the solvent and the components in the stevia leaves may produce harmful by-products, at least in a small amount [20]. Therefore, for this experiment, 3 solvents have been chosen to extract stevioside and Reb A namely, ethanol, acetone and water.

The objectives of this research are to find out the best extracting agent, to investigate how extraction time, temperature and solute: solvent ratio affect the yield of Reb A as well as stevioside and to determine the values of each parameter which produce the highest yield.

\section{METHODOLOGY}

\subsection{Materials \& Apparatus}

Table 1: List of materials and apparatus

\begin{tabular}{|c|c|}
\hline Materials & Apparatus \\
\hline Dried stevia leaves & Centrifuge machine \\
\hline 0.1M phosphoric acid & Conical flask \\
\hline Acetone & Heavy duty grinder \\
\hline Whatman No 1 filter paper (110mm) & HPLC machine \\
\hline Acetonitrile (HPLC grade) & Incubation shaker \\
\hline Ethanol absolute & Oven \\
\hline Distilled water & Sieve set \\
\hline Reb A standard & Vacuum filtration apparatus \\
\hline
\end{tabular}

\subsection{Sample Preparation and Extraction}

$100 \mathrm{~g}$ of fresh mature stevia leaves were dried in an oven to remove moisture in them [4]. Then, the leaves were grinded to powder form in a heavy duty grinder and sieved using $0.5 \mathrm{~mm}, 0.2 \mathrm{~mm}$ and $0.1 \mathrm{~mm}$ sieves. The fine powder was kept in sealed polyethylene bags and placed in a freezer at $4^{0} \mathrm{C}[12] .2 \mathrm{~g}$ of stevia powder was weighed using microbalance and placed in $1550 \mathrm{ml}$ conical flasks. Water, ethanol and acetone were poured into the conical flasks according to solute: solvent ratio of 1:5, 1:10, 1:15, 1:20 and 1:25 [1]. All 15 conical flasks were covered with aluminum foil and placed in incubation shaker. The temperature in the incubation shaker was set to $40^{\circ} \mathrm{C}$ and let to run at $150 \mathrm{rpm}$ for 2 hours. This set of extraction studied the material ratio. The second set of extraction was done with different extraction times and proceeded with another set for different temperatures. 
Table 2: Values of parameters investigated in extraction

\begin{tabular}{|c|c|c|c|}
\hline \multirow[t]{2}{*}{ Ratio } & \multicolumn{3}{|c|}{ Yield of reb A using respected extracting solvent } \\
\hline & Ethanol & Acetone & Water \\
\hline \multicolumn{4}{|l|}{$1: 5$} \\
\hline \multicolumn{4}{|l|}{$1: 10$} \\
\hline \multicolumn{4}{|l|}{$1: 15$} \\
\hline \multicolumn{4}{|l|}{$1: 20$} \\
\hline \multicolumn{4}{|l|}{$1: 25$} \\
\hline \multirow[t]{2}{*}{ Time (hours) } & \multicolumn{3}{|c|}{ Yield of reb A using respected extracting solvent } \\
\hline & Ethanol & Acetone & Water \\
\hline \multicolumn{4}{|l|}{1} \\
\hline \multicolumn{4}{|l|}{1.5} \\
\hline \multicolumn{4}{|l|}{2} \\
\hline \multicolumn{4}{|l|}{2.5} \\
\hline \multicolumn{4}{|l|}{3} \\
\hline \multirow[t]{2}{*}{ Temperature ${ }^{\circ} \mathrm{C}$} & \multicolumn{3}{|c|}{ Yield of reb A using respected extracting solvent } \\
\hline & Ethanol & Acetone & Water \\
\hline 40 & & & \\
\hline 45 & & & \\
\hline 50 & & & \\
\hline 55 & & & \\
\hline 60 & & & \\
\hline
\end{tabular}

\subsection{Centrifugation and Filtration}

After the extraction, the liquid phase containing the analyte was filtered from the solid phase by using Whatman No 1 filter paper (110mm). Then, the liquid phase was filled in separate centrifuge tubes and labelled. They were centrifuged at $1000 \mathrm{rpm}$ for 1 hour. After centrifugation, the liquid phase separated into supernatant and precipitate. Vacuum filtration was done to separate supernatant from precipitate. The supernatant was filled in separate test tubes, labelled and sealed properly to avoid contamination [22].

\subsection{HPLC Analysis}

HPLC analysis was done using Eclipse Plus C18 $(25 \mathrm{~cm} \times 4.6 \mathrm{~mm}$ I.D., $5 \mu \mathrm{m})$. The column temperature was kept at $27^{\circ} \mathrm{C}$ and $\mathrm{UV}$ detection was set at $210 \mathrm{~nm}$. The injection volume was fixed at $10 \mu \mathrm{L}$ with a flow rate of $1 \mathrm{ml} / \mathrm{min}$. HPLC column was equipoised by allowing mobile phase through it until a driftfree baseline was formed. The chromatograms of both sample and standard solutions were collected in 10 minutes. The chromatogram of each sample was compared to the standard to find the retention time 
of Reb A and stevioside. The peak areas (mAU*s) of Reb A and stevioside were calculated by a software called HPLC Offline [7].

Formula to calculate the percentage of Reb A and stevioside:

$$
\begin{aligned}
& \% \text { stevioside }=[\mathrm{Ws} / \mathrm{W}] \times[\mathrm{Aa} / \mathrm{As}] \times 100 \\
& \% \text { Reb } \mathrm{A}=[\mathrm{Ws} / \mathrm{W}] \times \mathrm{Ac} \times[1.20 / \mathrm{Ar}] \times 100
\end{aligned}
$$

where

Ws = mass $(\mathrm{mg})$ of stevioside in standard solution, $\mathrm{W}=$ mass of sample $(\mathrm{mg})$, Aa $=$ Peak area of stevioside from sample solution, As= Peak area of stevioside from standard solution, Ac $=$ Peak area of Reb A from sample solution, $1.20=$ relative molecular mass of Reb A, Ar = Peak area of Reb A from standard solution [14].

\section{RESULT \& DISCUSSION}

\subsection{Retention Time \& Calibration Curve}

Retention time is the time taken for solute to pass through a chromatography column. It includes the time from injection to detection. Calibration curve, also known as standard curve, is a general method for determining the concentration of a substance in an unknown sample by comparing the unknown to a set of standard samples of known concentration [4]. After obtaining the values of area under the peak of five concentrations of Reb A standard, a plot of peak area as a function of analyte concentration was developed and the linear regression was calculated by method of least squares. The retention time and correlation coefficient for stevioside were 2.237 minutes and 0.998 respectively whereas the retention time and correlation coefficient for Reb A were 2.183 minutes and 0.999 respectively. Figure 1 and 2 show the standard curve for stevioside and Reb A respectively.

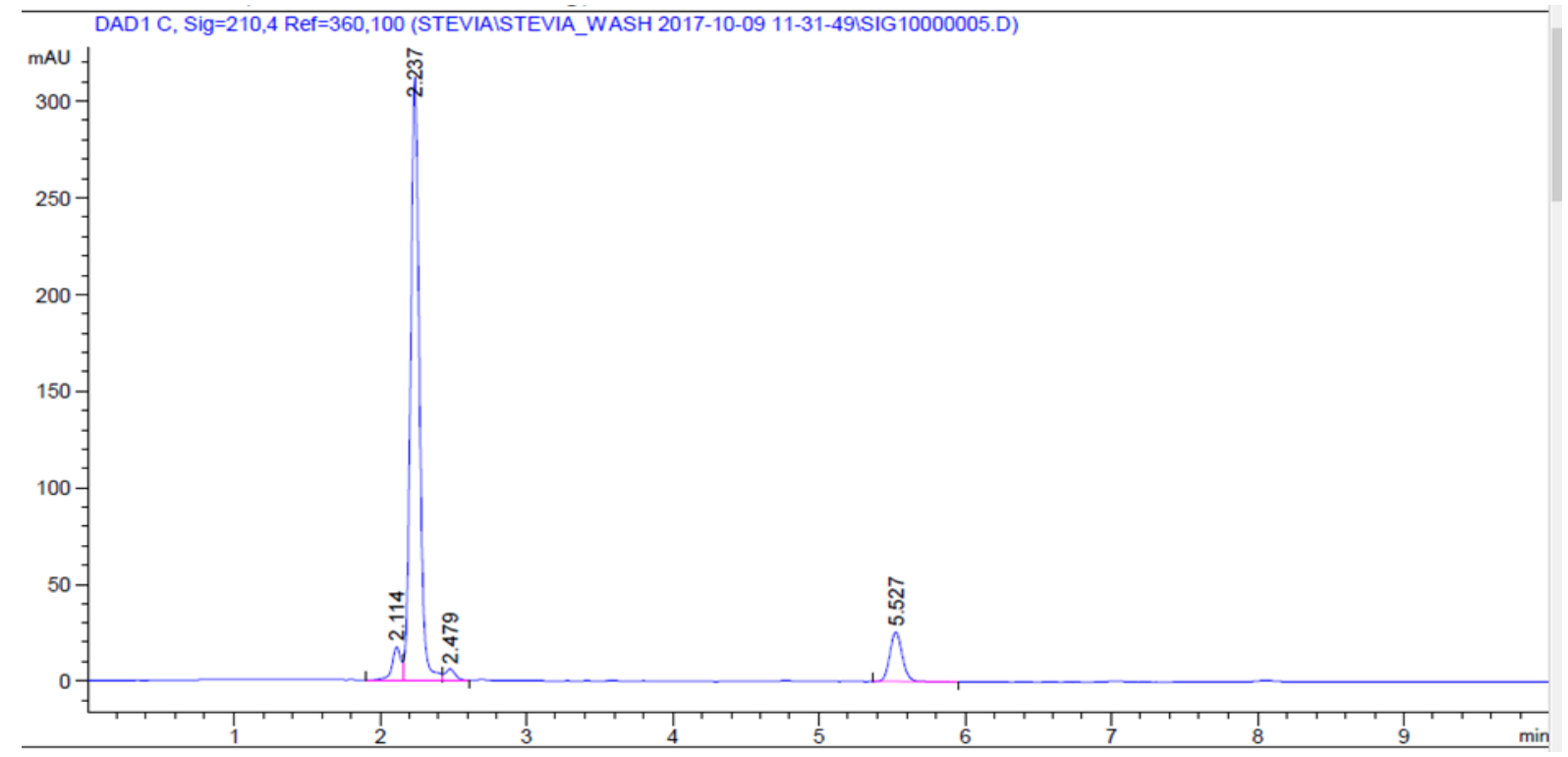

Figure 1: Standard curve for stevioside 


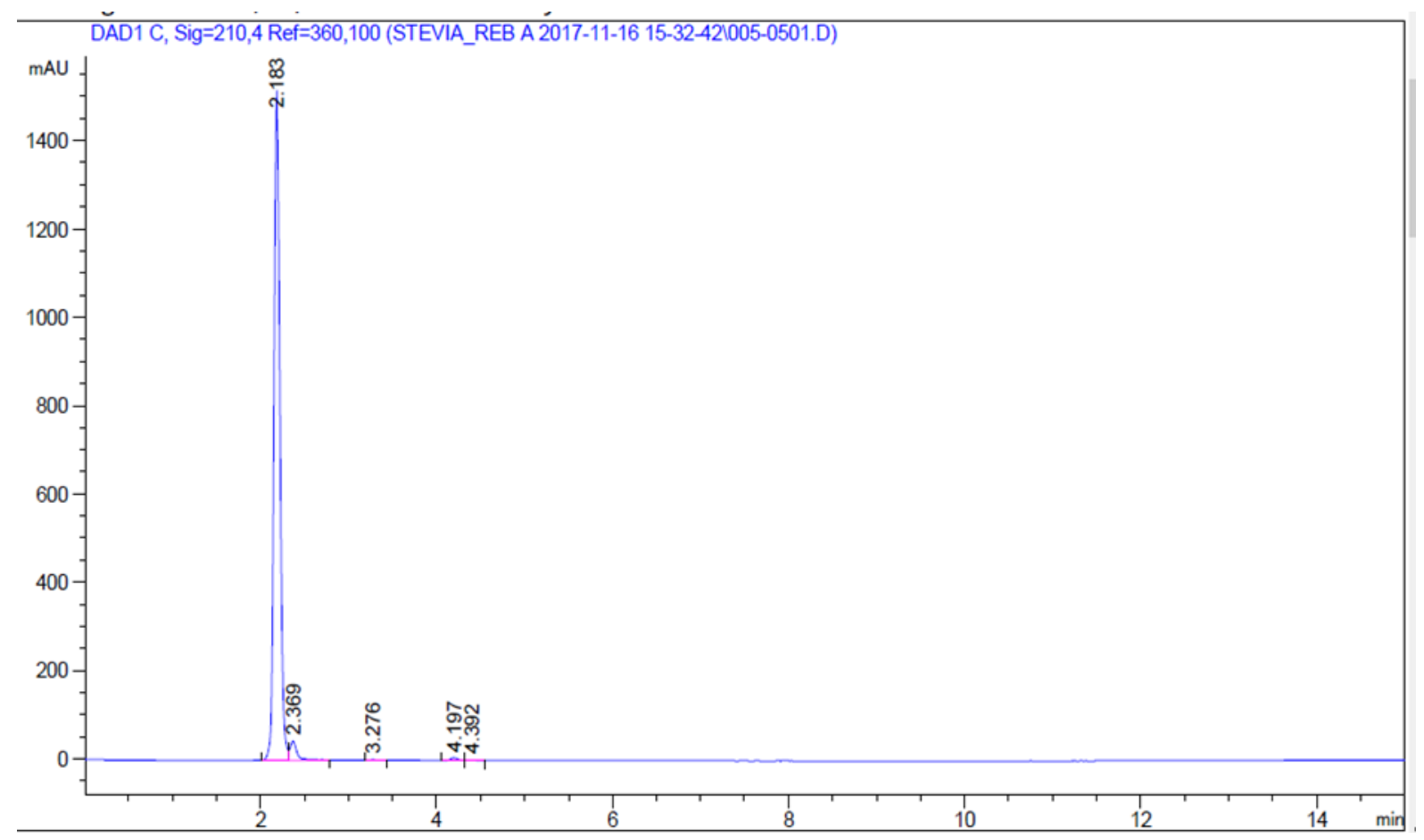

Figure 2: Standard curve for Reb A

\subsection{Effect of Material Ratio on Yield of Reb A \& Stevioside}

Overall, the yield of Reb A in ethanol extraction is quite low compared to stevioside. This is because the constituent of Reb A is only 2 to $4 \%$. Yield of Reb A is not affected much by the ratio between stevia powder and ethanol. Increase of ratio from 1:5 to 1:15 showed a slight decrease in the yield of Reb A but a further increase of ratio showed constant increment that lead to the highest yield of Reb A recovered, $0.45 \%$. Due to little amount of Reb A in the leaves, $10 \mathrm{ml}$ of ethanol was enough to extract the whole Reb A [8]. For stevioside, the yield increased with the material ratio. This is because more ethanol extracted more stevioside. However, when the ratio was set at 1:25, the stevioside yield started to decrease due to saturation of extract in the solution [8].

In acetone extraction, yield of Reb A increased as the ratio between stevia and acetone is increased from 1:5 to 1:10. Further increase in ratio caused the yield of Reb A to decrease because it is fully extracted and excess solvent will dissolve the extract and make the solvent saturated. Therefore, the best ratio to extract Reb A using acetone is 1:10. Highest yield for stevioside obtained from acetone extraction was $2.68 \%$ at a material ratio of 1:10. Further increase in the ratio decreased the yield of stevioside. Extraction of Reb A using water is not very good because traces of Reb A only appeared when extracted under ratio of 1:5. Increase of water results in disappearance of Reb A. This is due to dilution of Reb A in the solvent and cannot be quantified. So, it has been proven that the most preferable ratio when extracting Reb A is 1:5. The yield of stevioside increased with the ratio but only until 1:20. At a ratio of 1:25, the yield started to decrease because of saturation. Figure 3 shows the yield of Reb A and stevioside from ethanol, acetone and water extraction in different material ratio. 
Kalaiarasan Muthusamy, Mimi Sakinah Abdul Munaim /International Journal of Engineering Technology and Sciences 6:1 (2019) 120-130
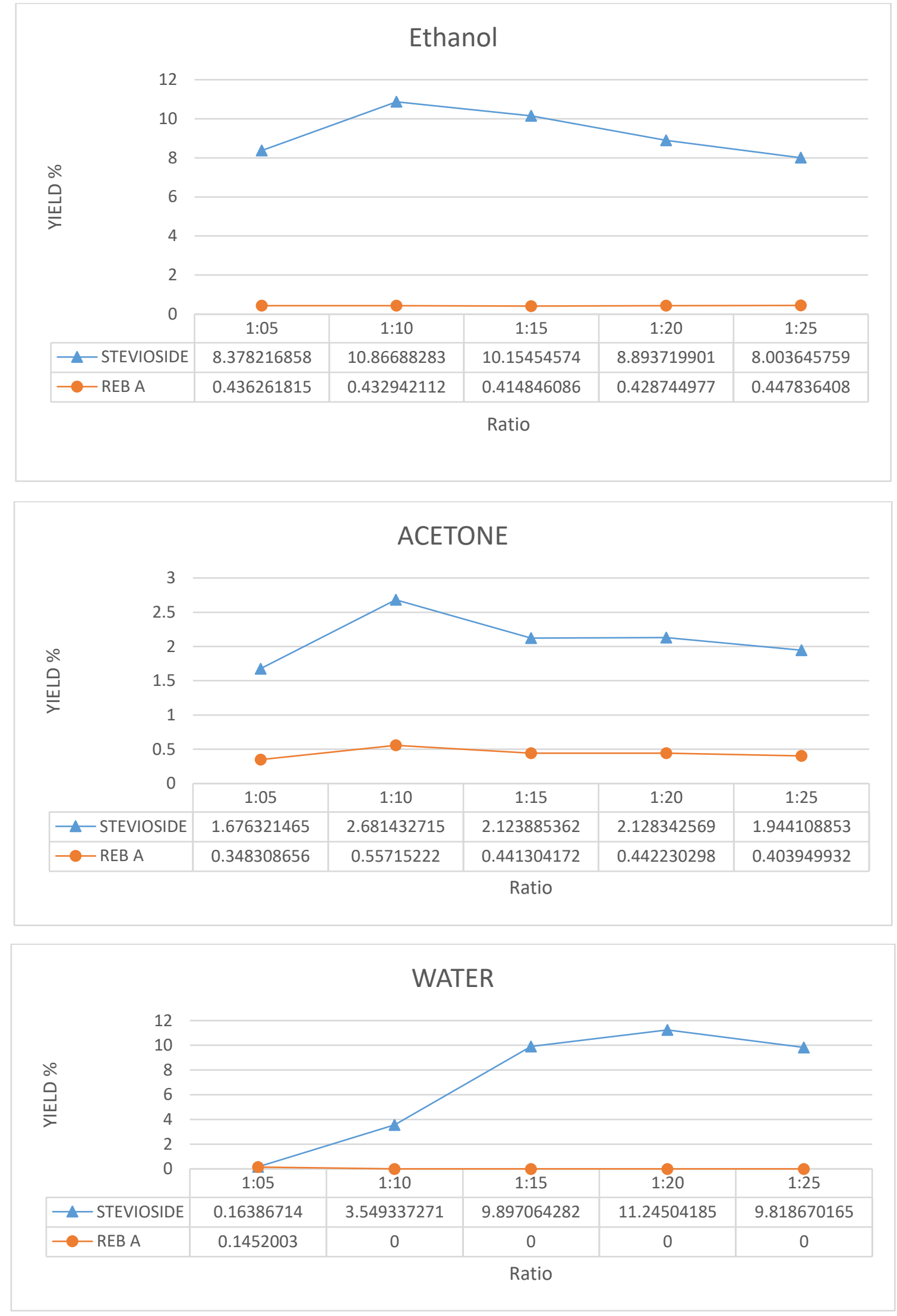

Figure 3: Yield of Reb A and stevioside from ethanol, acetone and water extraction in different material ratio 


\subsection{Effect of Extraction Time on Yield of Reb A \& Stevioside}

During ethanol extraction, highest yield of Reb A was achieved when extraction was conducted for 1 hour. Increase in extraction time decreased the yield of Reb A. Reb A can be oxidized easily in ethanol thus increase in extraction time will cause loss of Reb A [1]. In contrast, as the extraction time increased, the yield of stevioside increased gradually. Longer extraction time allows larger percentage of stevioside to be extracted.

The highest yield of Reb A in acetone extraction was obtained from a 3 hours extraction. Reb A yield has increased after the extraction reached 2 hour point. Two hours was not enough to extract Reb A from stevia when using acetone. Thus, most preferable extraction time for acetone is 3 hours. Whereas, 1 hour of acetone extraction gave the highest yield of stevioside with yield value of $5.44 \%$. Further increase of time resulted in a zig-zag pattern yield.

For water extraction, the maximum yield of Reb A obtained was $0.67 \%$ during a 1 hour extraction. Increase in extraction time resulted in zero yield. This is highly due to solvent saturation issue [8]. Therefore, it can be concluded that the most suitable extraction time is 1 hour and the highest Reb A yield is $0.67 \%$. For stevioside, the yield value increased with time until the 2 hour mark producing a yield of $11.72 \%$. Further increase in time decreased the yield of stevioside. Figure 4 shows the yield of Reb A and stevioside from ethanol, acetone and water extraction in various extraction time.

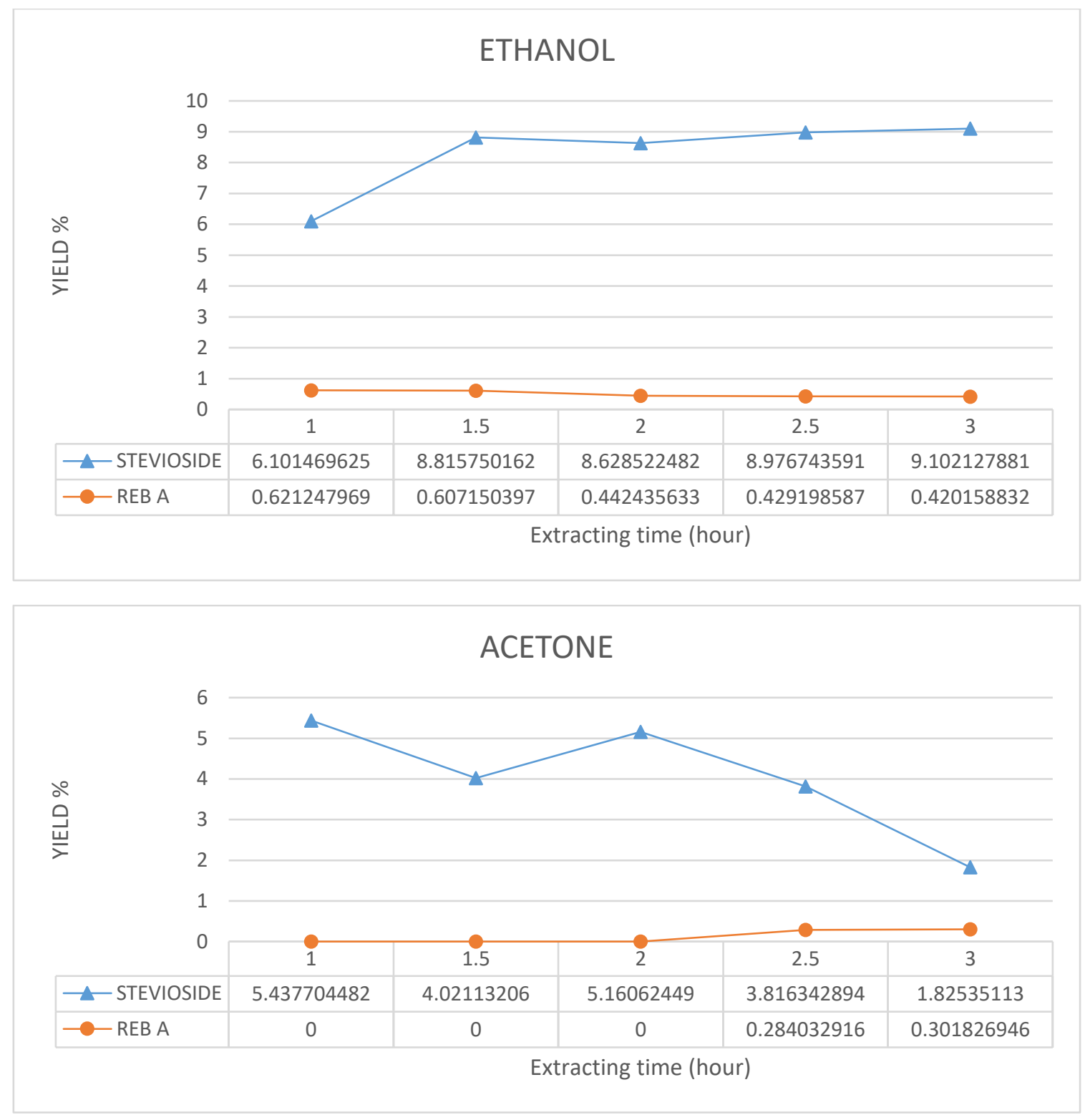




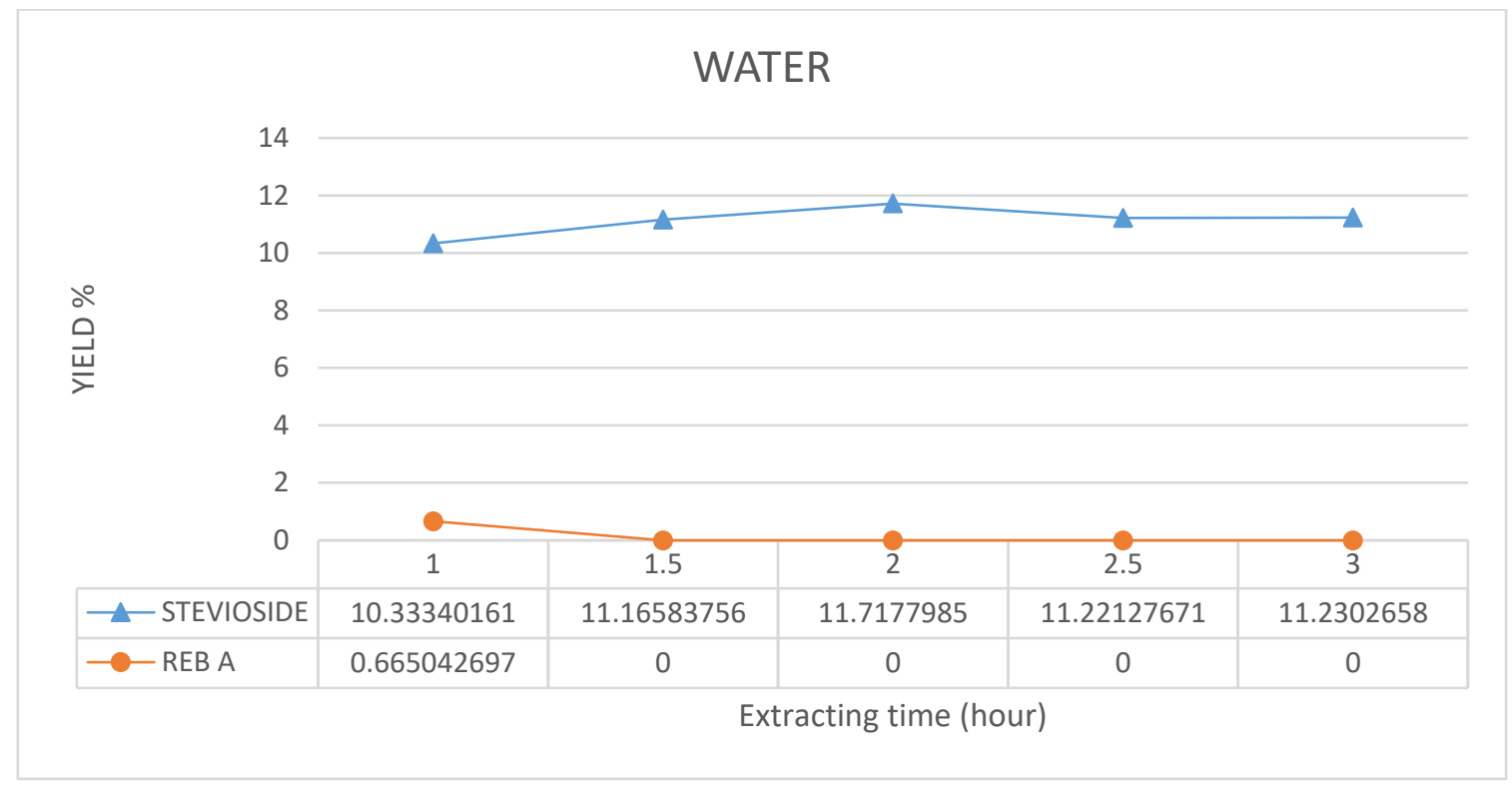

Figure 4: Yield of Reb A and stevioside from ethanol, acetone and water extraction in different extraction time

\subsection{Effect of Temperature on Yield of Reb A \& Stevioside}

Yield of Reb A was the highest when extracted using ethanol at $40^{\circ} \mathrm{C}$. Increase in temperature slightly decreased the yield of Reb A. High temperature can increase solvent flow thus improve the yield of Reb A but also decreases the fluid density that reduces the extraction ability [5]. Therefore, most preferred temperature for ethanol extraction is $40^{\circ} \mathrm{C}$. Also, at $40^{\circ} \mathrm{C}$, highest yield of stevioside was obtained.

Increase in temperature slightly triggered the recovery of Reb A in acetone extraction. For acetone, the best temperature to recover Reb A is $55^{\circ} \mathrm{C}$. Further increase in temperature caused decrease of yield. On the other hand, highest yield of stevioside was produced at $40^{\circ} \mathrm{C}$. Higher temperature resulted in lesser yield since the solvent was saturated with impurities which resulted from unwanted side reaction.

Highest yield of Reb A was obtained at $40^{\circ} \mathrm{C}(0.48 \%)$ in water extraction. Extraction at higher temperatures resulted no yield. This is due to low extraction efficiency of water towards Reb A. This indicates that the most optimum temperature for water extraction is $40^{\circ} \mathrm{C}$. Meanwhile, the yield of stevioside increased with temperature with the highest yield being $10.82 \%$. This shows that higher temperature will accelerate the extraction process. Figure 5 shows the yield of Reb A and stevioside during ethanol, acetone and water extraction in different temperature.

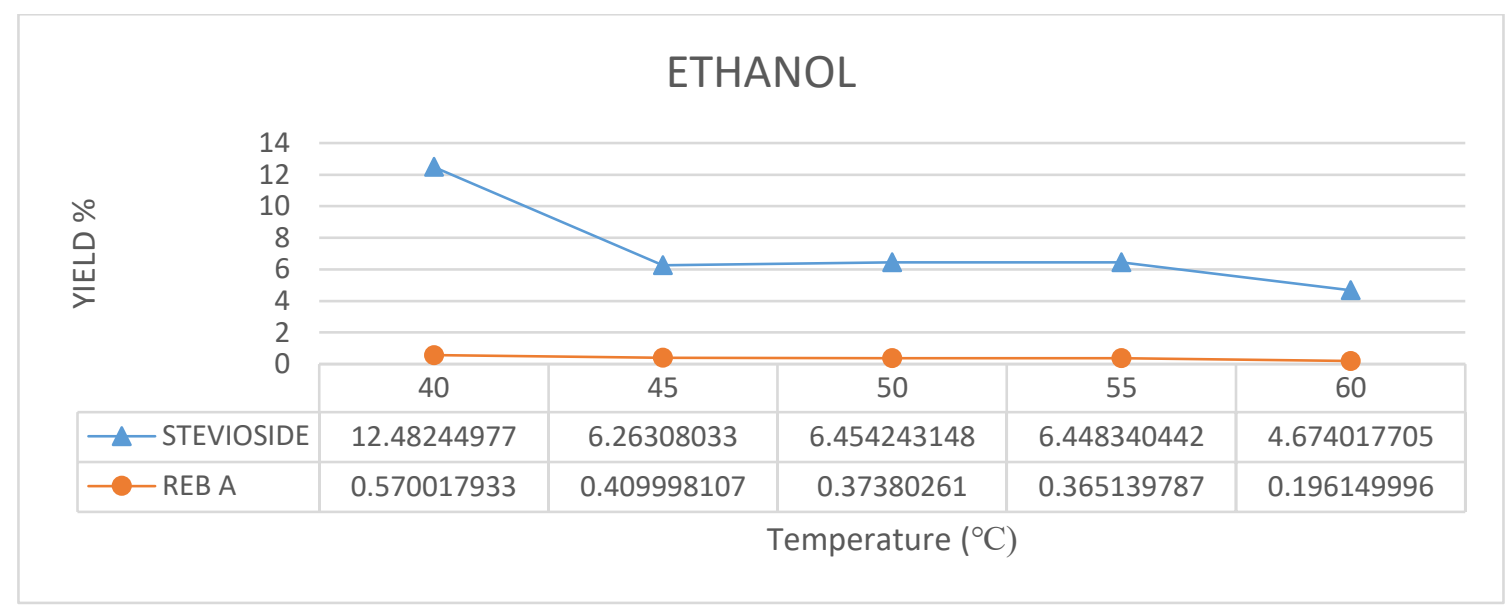




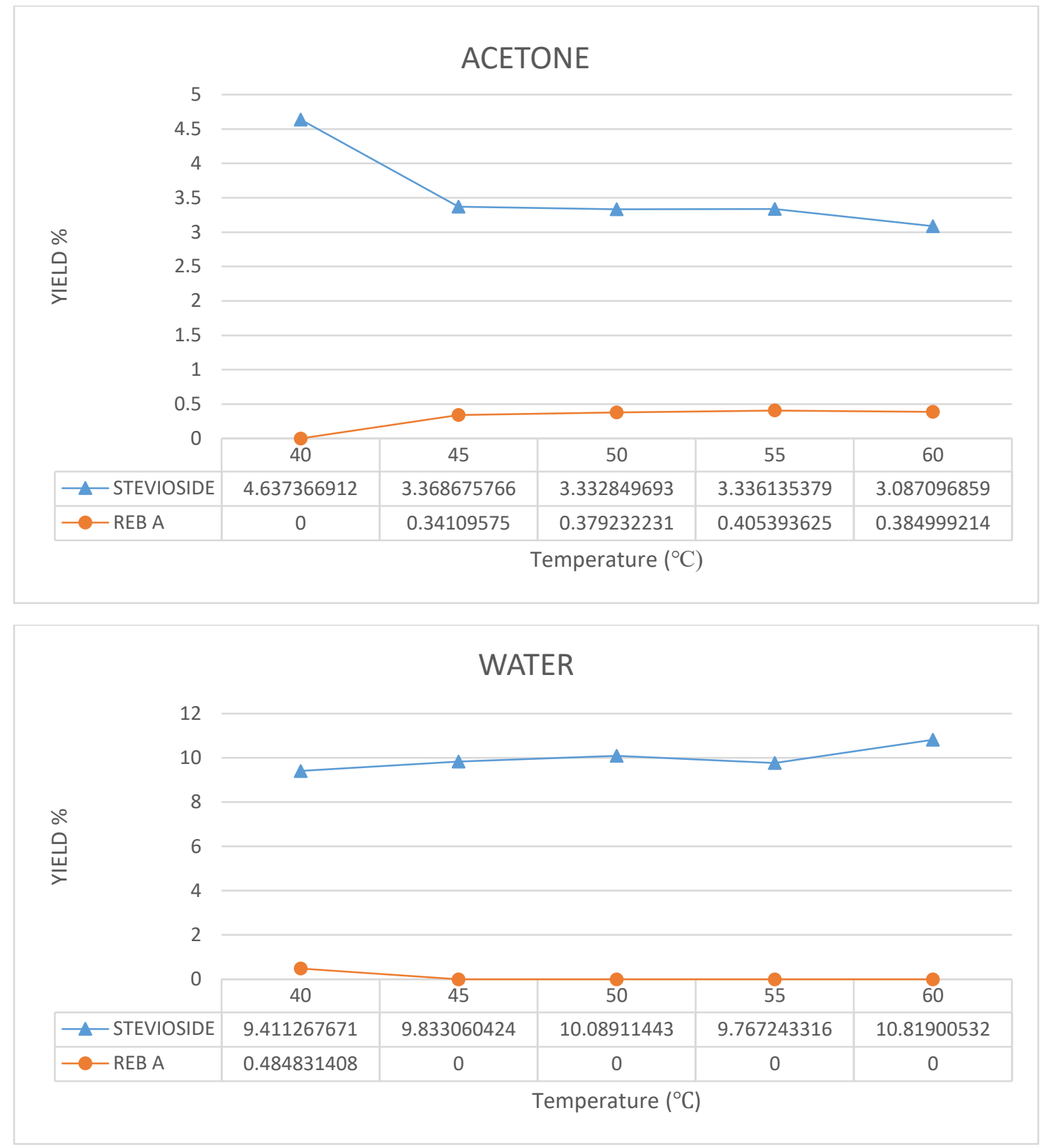

Figure 5: Yield of Reb A and stevioside during ethanol, acetone and water extraction in different temperature

\subsection{Comparison between Ethanol, Acetone \& Water}

Ethanol, acetone and water have been used to extract stevioside and Reb A. Best individual extraction parameters were obtained for each extracting solvent. For ethanol, the best parameter is stevia and solvent ratio of $1: 25,1$ hour of extraction in $40^{\circ} \mathrm{C}$. For acetone, the parameters are a ratio of 1:10, 3 hour of extraction in $55^{\circ} \mathrm{C}$. And lastly, the parameters for water are $1: 5,1$ hour and $40^{\circ} \mathrm{C}$. Ethanol extracted highest yield of Reb A $(0.57 \%)$ and stevioside (12.48\%) compared to acetone and water. This is because ethanol contains hydrophilic hydroxyl group. Since Reb A is a large and polar hydrocarbon molecule, it dissolves better in ethanol despite its high polar function [1]. 


\section{CONCLUSION}

All three extracting solvents were able to recover stevioside and Reb A. But, the best extracting agent is ethanol. Water can extract stevioside almost as much as ethanol but in term of extracting Reb A, the yield decreases with temperature. Meanwhile, acetone can extract Reb A not as much as ethanol but the yield of stevioside is very low. Besides determining the best extracting solvent, the yield of Reb A and stevioside can be improved by adjusting the ratio between stevia and solvent, extraction time and temperature. For ethanol extraction, the most preferable parameters are $1: 25,1$ hour and $40^{\circ} \mathrm{C}$.

\section{REFERENCES}

[1] Afandi, A., Sarijan, S., \& Shaha, R. K. "Optimization of Rebaudioside A Extraction from Stevia rebaudiana and Quantification by HPLC". Journal of Tropical Resources and Sustainable Science, 1(1), 62-70, 2015.

[2] Ahmad, M. "Stevioside and Stevia Sweetener in Food". Journal of Food Manufacturing, 5(14), 510$556,2018$.

[3] Devi, N. "An overview of diabetes types and treatments". Retrieved from: https://www. medicalnewstoday.com/articles/323627.php. Accessed on: $27^{\text {th }}$ September 2018.

[4] Gasmalla, A. A. "Extraction of Rebaudioside A by Sonication from Stevia Rebaudiana". Journal of Food Science, 5(9), 654-678, 2016.

[5] Guo-Qing, H., Hao-Ping, X., Qi-He, C., Ruan, H., Zhao-Yue, W., \& Traore, L. "Optimization of conditions for supercritical fluid extraction of flavonoids from hops (Humulus lupulus L.)". Journal of Zhejiang University SCI, 6(10), 999-1004, 2014.

[6] Kakigi, Y. "Classification of stevia sweeteners". Retrieved from: https://plants.usda.gov/ java/ClassificationServlet?source=display\&classid=STEVI. Accessed on: $23^{\text {rd }}$ October 2018.

[7] Kumari, N. "Sweet Compounds in Stevia". Journal of Pharmaceutics, 7(12), 456-479, 2016.

[8] Lemus-Mondaca, R. "Stevia rebaudiana Bertoni, source of a high-potency natural sweetener: A comprehensive review on the biochemical, nutritional and functional aspects". Food Chemistry, 7, 2016.

[9] Leung, A. Y., \& Foster, S. "Encyclopedia of common natural ingredients used in food, drugs and cosmetics (2nd ed.)". New York: John Wiley and Sons, 2, 478, 2014.

[10] Lewis, W. H. "Early uses of Stevia rebaudiana (Asteraceae) leaves as a sweetener in Paraguay". Econ. Bot, 46, 336-337, 2016.

[11] Li, Z. "Ultrasonic Extraction of Oil from Caesalpinia spinosa (Tara) Seeds". Journal of Chemistry, 16(1), 6-10, 2016.

[12] Martins, N. "Glycosides extraction”. Journal of Chinese Pharmaceuticals, 5(7), 210-258, 2016.

[13] Puri, M., Kaur, A., Barrow, C. B., \& Singh, R. S. "Citrus peel influences the production of an extracellular naringinase by Staphylococcus xylosus MAK2 in a stirred tank reactor". Applied Microbiology and Biotechnology, 89(2), 715-722, 2015.

[14] Rana, R. C. “Analysis of Steviosides”. Journal of Health Sciences, 45(7), 387-421, 2017.

[15] Rao, A. B. "Simple Extraction and Membrane Purification Process in Isolation of Steviosides with Improved Organoleptic Activity". Advances in Bioscience and Biotechnology, 7(8), 2015.

[16] Redfern, J. "Using Soxhlet Ethanol Extraction to Produce and Test Plant Material". Journal of Microbiology, 15(1), 45-46, 2016. 
[17] Richard, D. "Stevia rebaudiana Nature's Sweet Secret”. Vital Health Publishing, 9-15, 2016.

[18] Singh, V. "Cultivation of Stevia (Stevia rebaudiana): A Comprehensive Review". Advances in Agronomy, 8(12), 137-177, 2016.

[19] Soejarto, D. D., Compadre, C. M., Medon, P. J., Kamath, S. K., \& Kinghorn, A. D. "Potential sweetening agents of plant origin: Field search for sweet-tasting Stevia species". Economic Botany, 37(1), 71-79, 2015.

[20] Victor, M. "Separation and purification of Rebaudioside A". Retrieved from: https://www.jove.com/science-education/5538/solid-liquid-extraction. Accessed on: $20^{\text {th }}$ October 2018.

[21] Wade, L. G. "Studies on the optimal process to extract flavonoids from red-raspberry fruits". Nature and Science, 3(2), 43-46, 2017.

[22] Yang, R. "Simple Extraction of Steviosides". Journal of Food Chemistry, 6(8), 273-297, 2017. 